\title{
FDG PET/CT in carcinoma of unknown primary
}

\author{
Thomas C. Kwee • Sandip Basu • Gang Cheng • \\ Abass Alavi
}

Received: 31 July 2009 /Accepted: 23 September 2009/Published online: 31 October 2009

(C) The Author(s) 2009. This article is published with open access at Springerlink.com

\begin{abstract}
Carcinoma of unknown primary (CUP) is a heterogeneous group of metastatic malignancies in which a primary tumor could not be detected despite thorough diagnostic evaluation. Because of its high sensitivity for the detection of lesions, combined ${ }^{18} \mathrm{~F}$-fluoro-2-deoxyglucose positron emission tomography (FDG PET)/computed tomography (CT) may be an excellent alternative to $\mathrm{CT}$ alone and conventional magnetic resonance imaging in detecting the unknown primary tumor. This article will review the use, diagnostic performance, and utility of FDG PET/CT in CUP and will discuss challenges and future considerations in the diagnostic management of CUP.
\end{abstract}

Keywords ${ }^{18} \mathrm{~F}$-fluoro-2-deoxyglucose $\cdot$ Position emission tomography · Computed tomography · FDG PET/CT .

Carcinoma of unknown primary . Primary tumor detection

\section{Introduction}

Carcinoma of unknown primary (CUP) is defined as a biopsy-proven malignancy whose anatomical origin

\section{T. C. Kwee $(\square)$}

Department of Radiology, University Medical Center Utrecht, Heidelberglaan 100,

3584 CX Utrecht, The Netherlands

e-mail: thomaskwee@gmail.com

S. Basu $\cdot$ G. Cheng $\cdot$ A. Alavi

Division of Nuclear Medicine, Department of Radiology,

Hospital of the University of Pennsylvania,

Philadelphia, PA, USA

\section{S. Basu}

Radiation Medicine Center (Bhabha Atomic Research Center),

Tata Memorial Center Annexe,

Bombay, India remains unidentified after a thorough diagnostic evaluation. Such an evaluation should have included a detailed medical history, complete physical examination including pelvic and rectal examination, full blood count and biochemistry, urinalysis and stool occult blood testing, histopathological review of biopsy material with the use of immunohistochemistry, chest radiography, computed tomography (CT) of the abdomen and pelvis, and, in certain cases, mammography [1]. CUP is the seventh to eighth most frequently occurring cancer in the world and the fourth commonest cause of cancer-related death in both men and women [1]. CUP accounts for approximately $2.3-4.2 \%$ of cancer in both sexes. The annual age-adjusted incidence per 100,000 population in the USA is $7-12$ cases, in Australia 18-19 cases, and in the Netherlands 5.3-6.7 cases [1]. Obviously, the definition of CUP is arbitrary, and the development and application of more sophisticated diagnostic tests will decrease the incidence and prevalence of CUP. Patients with CUP are categorized into favorable and unfavorable subsets, according to number, location(s), and histology of metastases. Some favorable subsets require specific treatment approaches and have the potential for an excellent outcome [1]. However, in general, CUP follows an aggressive biological and clinical behavior, with a median survival ranging from only 2 to 10 months [1]. Nevertheless, it can be hypothesized that detection of a primary tumor may optimize treatment planning, which, in turn, may improve patient outcome. Indeed, some studies have shown that survival in patients in whom a primary tumor was eventually detected was higher than in patients in whom the primary tumor remained undetected $[2,3]$. Additional diagnostic procedures that can be used for primary tumor detection include a combination of various radiological, endoscopic, and serum tumor marker studies, depending on the specific signs and symptoms, histological 
results, and laboratory abnormalities [1]. However, these tests can be expensive, time-consuming, and invasive. Furthermore, in the majority of patients these tests may eventually fail to detect a primary tumor. Clearly, there is a need for an alternative, noninvasive imaging modality with a high diagnostic yield. In this context, combined positron emission tomography (PET)/CT, using the radiotracer ${ }^{18} \mathrm{~F}$ fluoro-2-deoxyglucose (FDG) may be an excellent problem-solving tool in patients with CUP. This article will review the use, diagnostic performance, and utility of FDG $\mathrm{PET} / \mathrm{CT}$ in CUP and will discuss challenges and future considerations in the diagnostic management of CUP.

\section{FDG PET/CT in CUP}

Why?

$\mathrm{CT}$ and magnetic resonance imaging (MRI) are two of the main technologies that came to dominate imaging of human disease in clinical practice. Major advantages of these techniques are their ability to provide cross-sectional images of the entire body, which is especially important in CUP, because the primary tumor can be located anywhere in the body. In addition, whole-body imaging may detect or exclude additional metastatic sites, which may have important therapeutic or prognostic consequences. However, CT and conventional MRI including (contrast-enhanced) T1-weighted and T2-weighted sequences are limited in that they basically only allow for the detection of anatomical abnormalities and abnormal contrast enhancement, as a result of which small lesions and non-enhancing lesions in normal-sized structures may be missed. This may in particular be an issue in CUP, in which the primary tumor may be of small size [1]. In addition, functional or metabolic pathological changes can occur in the absence of any corresponding anatomical changes and are not visualized by anatomical imaging at all. Furthermore, large amounts of image data are obtained in a single whole-body CT or MRI examination, as a result of which image interpretation is complex, time-consuming, and subtle pathological findings may be overlooked. Therefore, sensitivity of CT and anatomical MRI for the detection of the unknown primary tumor may be compromised. FDG PET does not have these drawbacks and may be an excellent alternative whole-body imaging modality in patients with CUP, as was already reported by Rege et al. [4] in 1994. The rationale for the use of the radiotracer FDG for PET imaging in CUP is the fact that the vast majority of malignant cancer phenotypes exhibit an increased glucose metabolism (Warburg effect) [5]. In contrast to CT and conventional MRI, FDG PET offers high lesion-to-background contrast, making it a potentially more sensitive imaging modality for the detection of lesions. For example, Roh et al. [6] have shown that sensitivity of FDG PET/CT $(87.5 \%)$ was significantly higher $(p=0.016)$ than that of CT alone $(43.7 \%)$ in detecting primary tumors in 44 patients presenting with cervical metastases from unknown origin. Similarly, in the study by Nassenstein et al. [7] who investigated 39 patients with cervical metastases of unknown origin CT alone revealed the primary tumor in only 5 patients $(13 \%)$, while FDG PET alone and combined FDG PET/CT detected a primary tumor in 10 patients $(26 \%)$ and 11 patients $(28 \%)$, respectively. In addition, in their series of 21 patients with cervical metastases of unknown origin, Freudenberg et al. [8] showed that CT alone detected only 5 primary tumors (23\%), while FDG PET alone and combined FDG PET/CT detected 11 primary tumors $(52 \%)$ and $12(57 \%)$ primary tumors, respectively, with a significant difference $(p=0.03)$ between CT alone and FDG PET/CT. Furthermore, Gutzeit et al. [9] reported that $\mathrm{CT}$ alone depicted a primary tumor in only 8 of 45 patients (18\%), while FDG PET alone and combined FDG PET/CT detected primary tumors in 11 patients (24\%) and 15 patients (33\%), respectively. Current commercially available PET systems can now reach a spatial resolution of about 4-7 $\mathrm{mm}$ for whole-body imaging $[5,10]$, but thanks to its high lesion-to-background contrast, lesions with a size below this spatial resolution may even be detectable.

\section{How to perform?}

In the majority of clinics combined PET/CT systems, equipped with 16- to 64-section multidetector row CT units, have replaced the stand-alone PET scanner [10]. The CT component of a combined PET/CT system offers wellrecognized advantages, such as more accurate localization of sites with FDG uptake, thereby reducing the problems of physiological FDG uptake being misinterpreted as pathological and false localization of disease and allowing for more accurate image-guided biopsy of suspected primary tumor sites. Furthermore, its use for attenuation correction of PET emission data provides low-noise attenuation correction factors, eliminates bias from emission contamination of postinjection transmission scans, and reduces whole-body FDG PET/CT examinations to 10 $\min$ or less [10]. Three studies [7-9] have directly compared FDG PET/CT to (CT-based attenuationcorrected) FDG PET alone in CUP. In all three studies [7-9], FDG PET/CT was able to detect a few more primary tumors than FDG PET alone, although these differences were not statistically significant. Nevertheless, a combined PET/CT system is favored above a stand-alone PET scanner in patients with CUP because of its previously mentioned advantages. 
For the FDG PET component of a FDG PET/CT study in patients with CUP, a standard protocol can be used [11]. Importantly, although the optimal time for data acquisition has not been determined yet in CUP, it may be recommended to perform (additional) delayed PET imaging (e.g., 3 or $4 \mathrm{~h}$ after FDG administration) in order to improve lesion-to-background contrast, as will be discussed in a later section. As will be outlined in a later section, the lung has been reported to represent one of the two main locations with the highest number of false-positive FDG PET/CT results in patients with CUP [12]. It has also been reported that focal intrapulmonary FDG uptake may occur as a result of iatrogenic pulmonary microembolism due to aspiration of blood during intravenous FDG administration [13], which should be avoided. The second main location of false-positive FDG PET/CT results is the oropharynx [12]. Although the risk of physiological FDG uptake being misinterpreted as a lesion cannot be completely eliminated, patients should be instructed to avoid or minimize talking, chewing, and swallowing immediately before and after FDG administration in order to minimize FDG uptake in the laryngeal and pharyngeal muscles [14].

Although data regarding the influence of different CT protocols on diagnostic performance of FDG PET/CT in CUP are largely lacking, it is recommended to use a collimation of less than $2.5 \mathrm{~mm}$, in order to detect small primary lung cancers [15]. Of note, lung cancer is the most frequently reported primary tumor in patients with CUP [12, 16-19]. While it may be argued that the use of intravenous and oral contrast agents may improve detectability of the primary tumor, neither the study of Fencl et al. [20] nor a recent metaanalysis [12] showed any beneficial effect of this approach on diagnostic performance in patients with CUP compared to using no contrast agents at all. Furthermore, because regions containing high concentrations of contrast medium may artificially lead to overestimation of FDG uptake when using CT-based attenuation correction [21], and because iodinated contrast agents may cause adverse reactions, including rarely occurring but life-threatening contrast-induced nephrotoxicity and anaphylactic shock [22], it may be preferred to perform a $\mathrm{CT}$ scan without contrast agents.

\section{How to interpret?}

The most frequently reported unknown primary tumor locations include the lung, pancreas, and oropharynx [12, 16-19]. Therefore, these locations should carefully be evaluated. Furthermore, histopathological diagnosis and metastatic pattern may suggest possible locations of the primary tumor. For example, in patients with involvement of the upper or mid-cervical lymph nodes, a primary site in the head and neck should be suspected [23]. In patients with poorly differentiated carcinoma and left supraclavicu- lar lymphadenopathy (Virchow's node), a primary gastrointestinal tumor may be detected [23]. In women with axillary lymph node metastases, primary breast cancer may be suspected. On the other hand, women with peritoneal carcinomatosis may have primary ovarian cancer [23]. Furthermore, in men with osteoblastic bone metastases, prostate cancer may be the primary tumor [23]. Nevertheless, the primary tumor can be located anywhere in the body, and the entire body should be carefully reviewed for lesions, including previously undetected additional metastases.

Finally, special attention should be paid to the evaluation of the oropharynx and lung, because these are the locations with the most frequently reported false-positive FDG PET/ CT results [12] (Figs. 1 and 2). The oropharynx is a difficult area to evaluate; physiological FDG uptake in the lymphoid tissue of the adenoids and Waldeyer's ring, and overlap between tumor and physiologic FDG uptake, may impair diagnostic performance [24]. Nevertheless, accurate localization of increased FDG uptake using CT images may, in part, reduce the rate of false-positive results (e.g., recognition of physiological muscle FDG uptake). Furthermore, although focally increased FDG uptake may resemble a primary lung cancer, its CT pattern may indicate the presence of a benign inflammatory or infectious lesion (e.g., pneumonias usually present with a lobular, segmental, or lobar pattern on CT), or a pulmonary embolism or infarction (e.g., a wedgeshaped peripheral region of consolidation on CT should raise the possibility of a pulmonary infarction) [25-27].

\section{Diagnostic yield}

It should be realized that despite extensive work-up, less than $20 \%$ of patients with CUP have a primary site of their cancer identified antemortem. In addition, autopsy studies have reported that $70 \%$ of cases remained undiagnosed [1], which well reflects the diagnostic challenge of currently available imaging modalities. It has still not been clarified why the majority of primary tumors cannot be detected. Common hypotheses include spontaneous regression or immune-mediated destruction of the primary tumor or the inherent small size of the primary tumor (metastatic spread is favored above local tumor growth) [1, 28, 29].

Several meta-analyses have assessed the diagnostic performance of stand-alone FDG PET in patients with CUP [30-32], reporting primary tumor detection rates of $41 \%$ [30], 24.5\% [31], and 43\% [32], with a generally higher sensitivity than specificity using histopathological analysis of tissue obtained by biopsy or surgery and/or other imaging procedures or clinical follow-up as the standard of reference [30-32]. However, as mentioned previously, combined PET/CT systems have many advantages over the stand-alone PET scanner and are rapidly replacing the latter. One recent meta-analysis investigated 

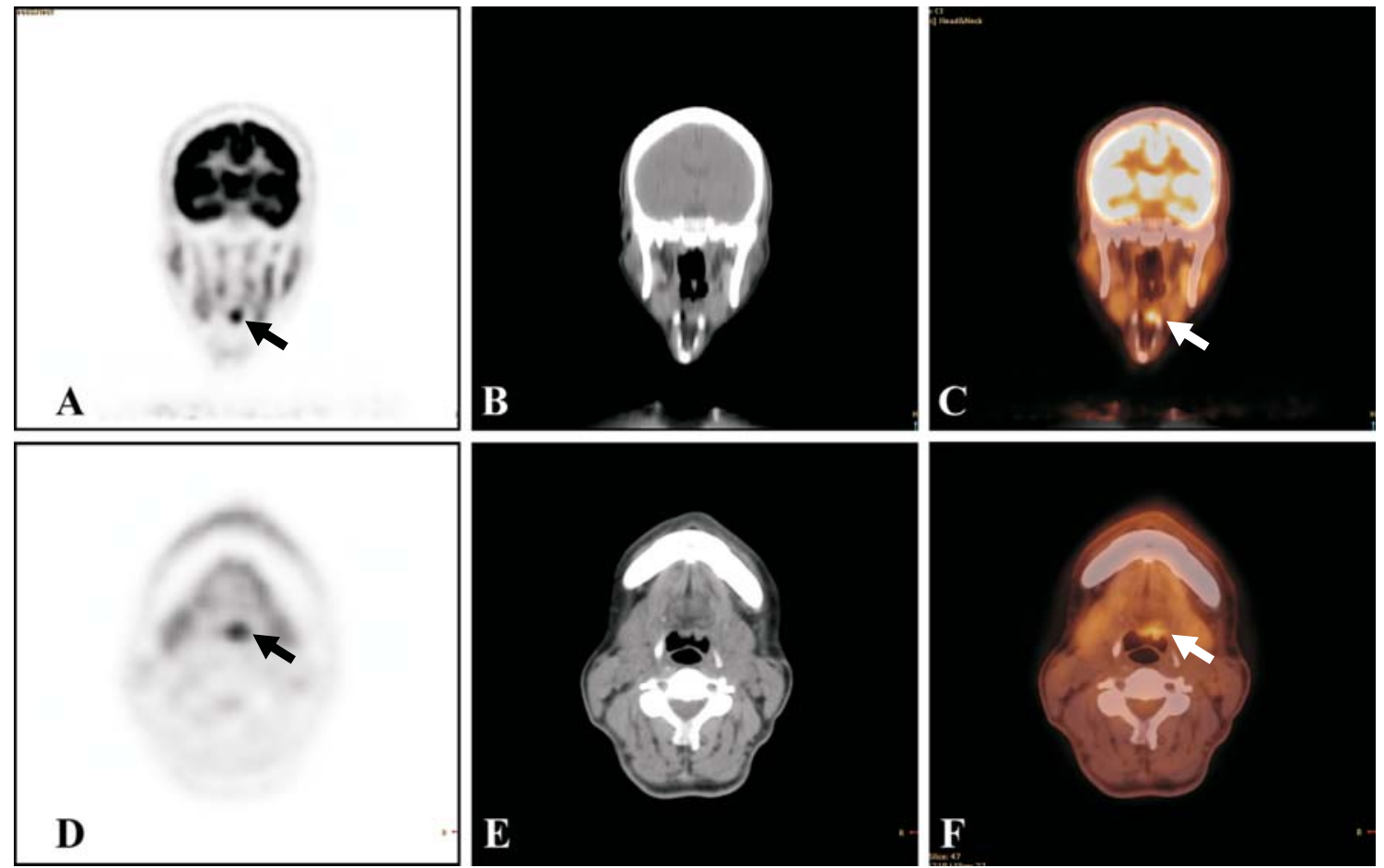

Fig. 1 FDG PET/CT in a 52-year-old man who presented with a pathologically enlarged left cervical lymph node. Excision biopsy of the lymph node and subsequent histopathological examination indicated metastatic squamous cell carcinoma of unknown primary. Microdirect laryngoscopy and bronchoscopy were performed with (blind) biopsy and tonsillectomy; subsequent histopathological examination only showed reactive changes in the tonsils, pyriform sinus, and left tongue base, without any indication for the presence of primary tumor. FDG PET in the coronal (a) and axial plane (b) shows intense FDG uptake in the left supraglottic region (arrows), likely representing the primary tumor. Corresponding CT and fused FDG $\mathrm{PET} / \mathrm{CT}$ images in the coronal (b, c, respectively) and axial plane (e, f, respectively) localize the lesion at the base of the tongue (arrows). Histopathological examination of a directed biopsy of the lesion revealed in situ squamous cell carcinoma of the tongue base (moderately to poorly differentiated) the diagnostic performance of FDG PET/CT and reported a primary tumor detection rate of $37 \%$ [12]. Diagnostic performance of FDG PET/CT was not significantly different between patients presenting with cervical metastases and patients presenting with extracervical metastases, while lung, oropharyngeal, and pancreatic cancer were reported to represent the most frequently detected primary tumors (Fig. 3) [12]. Lung and pancreatic cancer are indeed also the most common primary tumor locations in autopsy studies [16-19]. The rather unexpected high rate of primary oropharyngeal cancers in the aforementioned meta-analysis [12] can be explained by the fact that it included a large proportion of patients with cervical metastases, whose primary tumors are most frequently located in the oropharynx [33]. On the other hand, breast cancer appears to be the most common cause of a false-negative FDG PET/CT result (Fig. 4) [12]. This is due to the well-documented inability of FDG PET(/CT) to consistently demonstrate small $(<1.0 \mathrm{~cm})$ and low-grade lesions with low or no FDG uptake (e.g., tubular carcinoma and noninvasive cancers such as ductal or lobular carcinoma in situ) [34]. This limitation of FDG PET/CT should be kept in mind and in case of a high suspicion of a primary breast cancer (e.g., in case of axillary lymph node metastases) [23], a dedicated MRI scan may be considered [35, 36]. Similar to that in the breast, false-negative FDG PET/CT results in other locations are most likely attributable to small lesion size and low or no FDG uptake. In contrast, the oropharynx and the lung are the two most common locations of false-positive FDG PET/CT results [12] (Fig. 5). Literature on the exact causes of falsepositive FDG PET/CT results is scarce, although benign inflammatory lesions [7, 9, 37] and pulmonary infarction [9] have been reported etiologies. Furthermore, of special note is the fact that incidental pulmonary emboli have been reported to occur in $4 \%$ of patients with cancer [38]. It can be speculated that this may, in part, explain the rate of falsepositive FDG PET/CT results in the lung. Figures 1 and 2 show two examples in which FDG PET/CT was able to detect the unknown primary tumor.

Is it worthwhile?

Although it is widely accepted that CUP is a heterogeneous group of metastatic malignancies, the true nature of this 

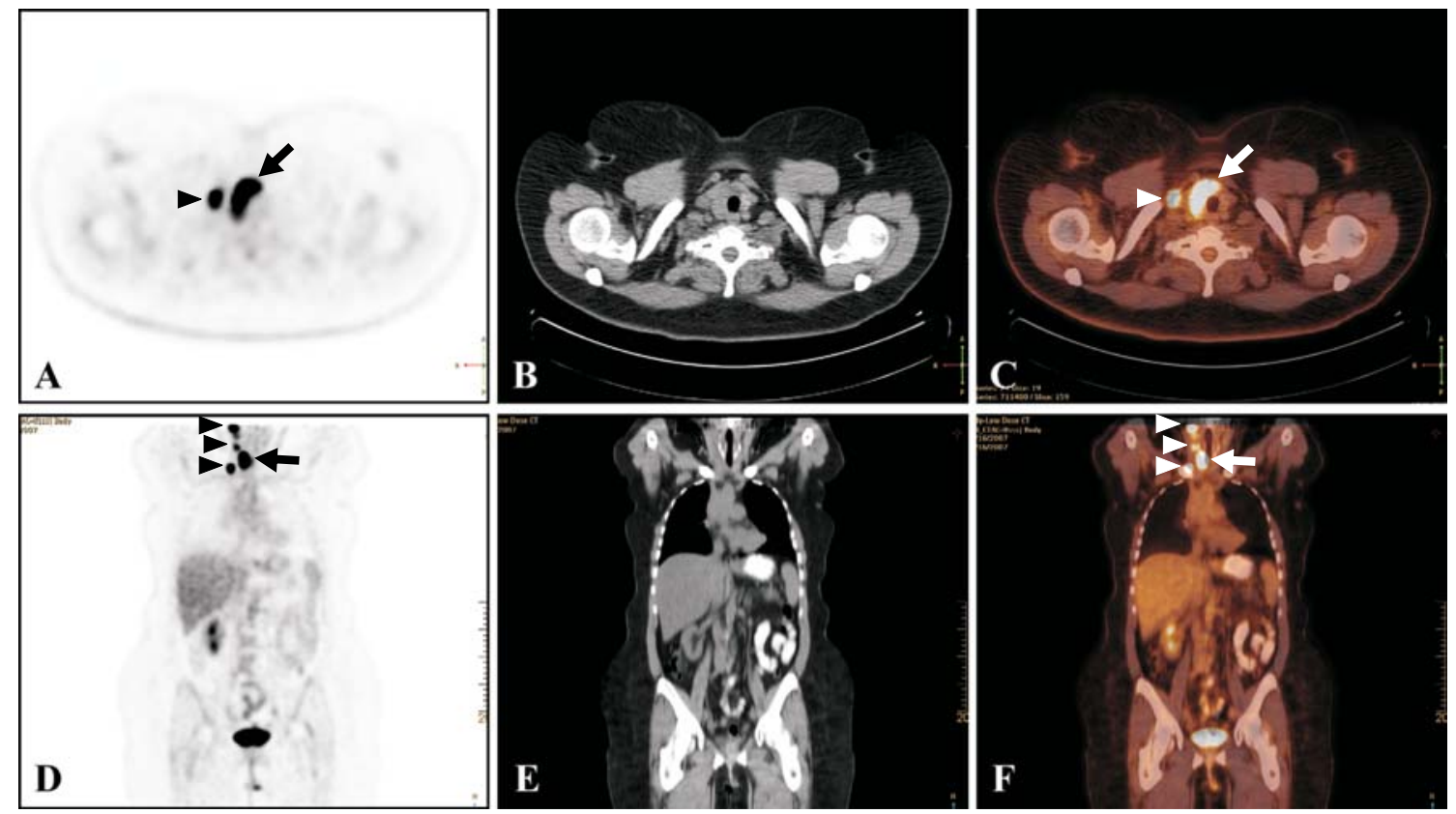

Fig. 2 FDG PET/CT in a 61-year-old woman who presented with a right neck mass. Neck CT confirmed the pathologically enlarged right cervical lymph node, showed slight asymmetry at the right tongue base and oropharynx, and an enlarged, heterogeneous right lobe of the thyroid gland. Fine-needle biopsy and subsequent histopathological examination of the pathologically enlarged right cervical lymph node indicated metastatic carcinoma of unknown primary. Tumor cells were positive for CK7, focally positive for TFF1, and negative for calcitonin and thyroglobulin, suggesting the possibility of a primary

clinical entity is still unknown. CUP may be considered as metastatic disease in patients in whom the primary tumor has not been found and which did not result in clinical signs of disease $[28,29]$. If this is the case, detection of the lung tumor. FDG PET in the axial (a) and coronal plane (d) shows intense FDG uptake of the thyroid (arrows), likely representing the primary tumor, and intense FDG uptake in the right cervical, supraclavicular, and paratracheal lymph nodes. Corresponding CT and fused FDG PET/CT images in the axial (b, c, respectively) and coronal plane (e, $\mathbf{f}$, respectively) confirm the localization of the lesions seen at FDG PET (arrows). Fine-needle aspiration of the thyroid and subsequent histopathological examination revealed a primary papillary thyroid carcinoma

primary tumor is worthwhile, because it may lead to more specific treatment planning and improve outcome [28, 29]. On the other hand, CUP may represent a separate group of cancers with genetic and phenotypic characteristics that
Fig. 3 Bar graph showing percentages of primary tumors detected by FDG PET/CT per location [12]

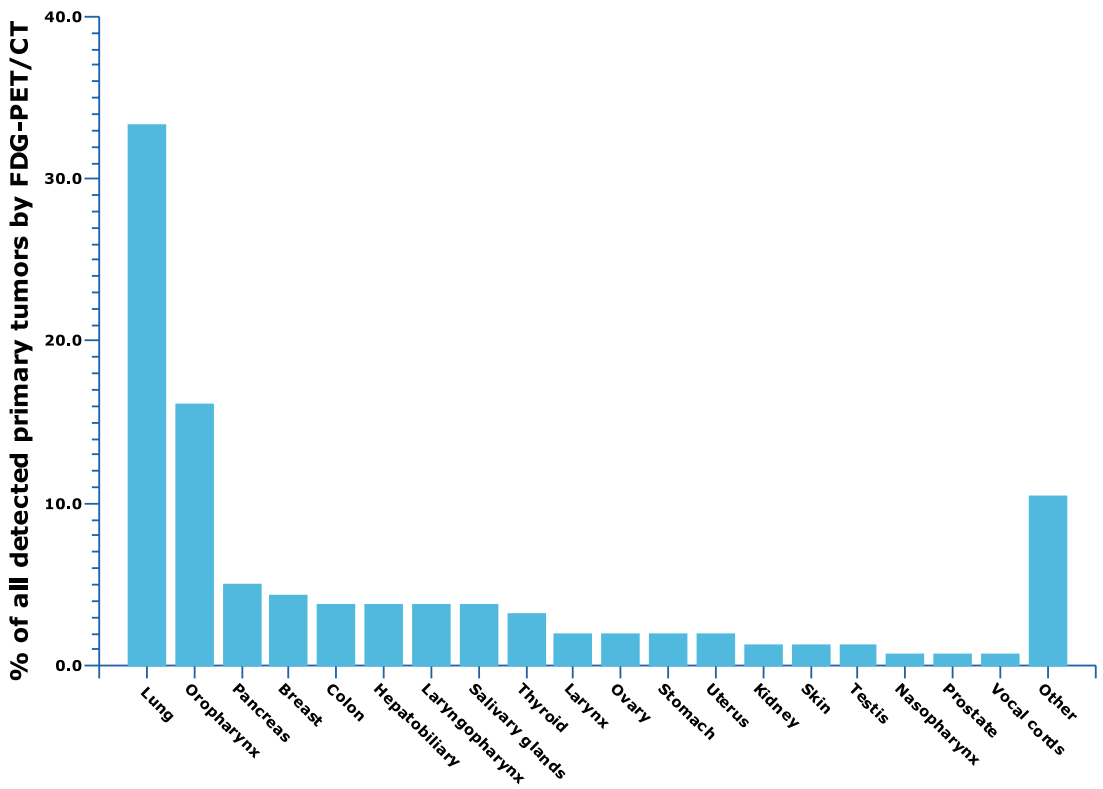


Fig. 4 Bar graph showing percentages of false-negative FDG PET/CT results per location [12]

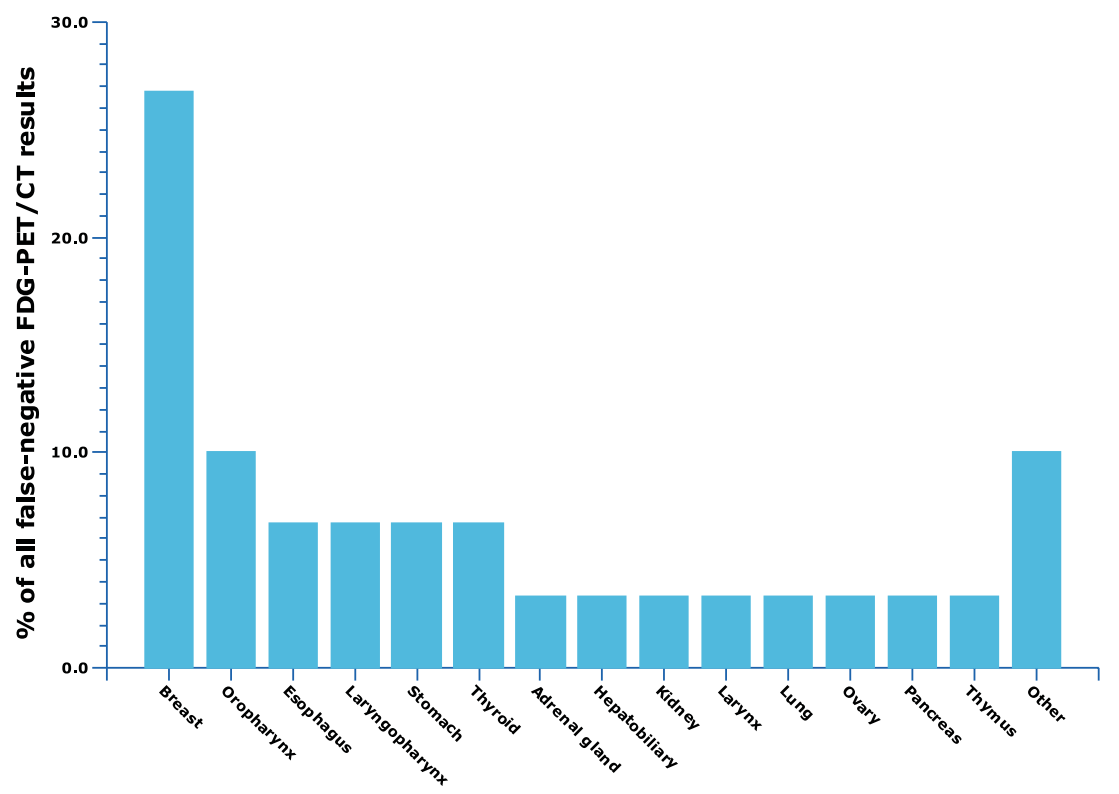

underlie their unique clinical presentation $[28,29]$. In this case, detection of the primary tumor is of minor importance, and diagnostic evaluation should focus on the identification of treatable subsets [28, 29]. In other words, research should focus on the metastatic genotype and phenotype and on the detection of specific biochemical or molecular targets for the development of CUP-specific therapy $[28,29]$. It should also be realized that FDG PET/ $\mathrm{CT}$ is an expensive examination, and false-positive FDG $\mathrm{PET} / \mathrm{CT}$ findings may result in unnecessary additional invasive diagnostic procedures, which have associated morbidities and costs [39]. Furthermore, the majority of unknown primary tumors are not detected, either due to insufficient sensitivity of FDG PET/CT or due to disappearance of the primary tumor [12].

Regardless of its ability to detect an unknown primary tumor, another important aspect of FDG PET/CT that should be considered is its ability to identify or rule out additional metastatic sites, which may have important implications for patient management. This may particularly be of interest in patients with CUP who present with lymph node metastatic disease only, because $M$ stage, which has important therapeutic and prognostic consequences, is still unknown in these patients. Also, detection of additional
Fig. 5 Bar graph showing percentages of false-positive FDG PET/CT results per location [12]

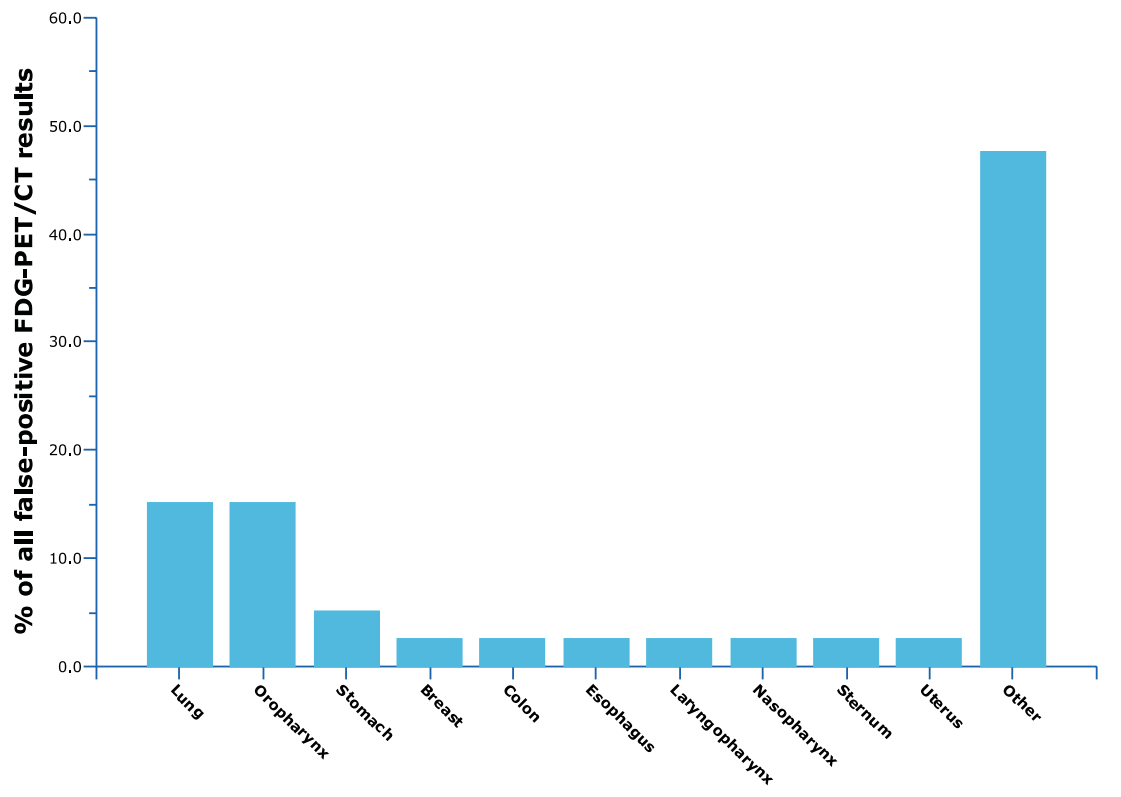


lymph node involvement based upon the FDG PET/CT study might help in modifying and planning the radiotherapy field appropriately, which is the pivotal modality of therapy in these patients. Furthermore, a baseline FDG PET/CT may also play a valuable role for treatment monitoring following therapeutic intervention [40].

Despite the limited evidence regarding its utility, a multidisciplinary expert panel of oncologists, radiologists, and nuclear physicians with expertise in PET/CT concluded that using FDG PET(/CT) in the diagnostic work-up of patients with CUP cancer is beneficial [41]. If FDG PET(/CT) findings are positive, confirmatory biopsy is necessary because of the risk of false-positive results [41]. Furthermore, the expert panel recommended that if FDG PET(/CT) findings are negative in case of a suspected unknown primary tumor in the head and neck region, further effort should be made to identify the primary tumor because of the chance of false-negative results [41]. However, CUP is not yet among the nine oncology types that are currently reimbursed by Medicare. Nevertheless, under Medicare's Coverage with Evidence Development (CED) policy, FDG PET(/CT) became a covered service for previously noncovered cancer indications, including CUP, if prospective registry data were collected [42]. Recently reported data from the National Oncologic PET Registry (NOPR) showed that 1,929 of $22,976(8.4 \%)$ FDG PET(/CT) scans under the CED policy were performed for diagnosis in patients with CUP [42]. Although changes in patient management due to FDG PET(/CT) findings were not reported for CUP separately, it was stated that FDG PET/CT changed treatment decisions in $36.5 \%$ of all cases after FDG PET(/CT), while the impact of FDG PET(/CT) on treatment decisions varied minimally by the specific cancer indication [42]. In addition, four individual studies in patients with CUP reported the therapeutic impact of FDG PET/CT; in these four studies, FDG PET/CT modified therapy in $18.2-60 \%$ of patients [43-46]. Furthermore, one study [37] reported that median overall survival between FDG PET/CT-positive and FDG PET/CT-negative groups was not significantly different, while another study [20] reported that the survival rate of CUP patients with at least one hypermetabolic lesion was significantly lower $(p<0.0279)$ than that of the remaining CUP patients. However, to the best of our knowledge, no study has investigated FDG PET/CT-modified patient outcomes. Therefore, the additional value of FDG PET/CT to patients with CUP and its cost-effectiveness should be further investigated.

\section{Challenges and future considerations}

Compared to other diagnostic procedures that are often used in patients with CUP (e.g., CT alone and endoscopic procedures), FDG PET/CT is both noninvasive and a very sensitive tomographic whole-body imaging modality, allowing for the detection of a primary tumor and complete tumor staging in a single examination. It can be argued that if FDG PET/CT fails to detect a primary tumor, other diagnostic procedures are also likely to fail. Therefore, perhaps FDG PET/CT should be used as a first-line imaging modality in all patients with metastatic disease rather than using it after other diagnostic procedures have failed to identify a primary tumor. This possible beneficial shift in the diagnostic work-up of patients with metastatic disease requires further investigation.

In CUP the primary tumor is often of small size [1]. Small tumors with a size around or below the spatial resolution of a PET system may not be reliably detected unless intense FDG uptake is present. However, even if such a small lesion has a high FDG uptake, partial volume averaging with the surrounding normal tissue may occur, resulting in a considerable decrease in the qualitatively or (semi-)quantitatively perceived FDG uptake. Consequently, the lesion may incorrectly resemble a benign process, which generally has a lower FDG uptake [standardized uptake value (SUV) $<2.5-3.0]$ than a malignant lesion [11]. Therefore, technological advances that lead to a higher signal to noise ratio (SNR) and a higher spatial resolution are likely to increase the diagnostic yield of FDG PET/CT in CUP. In this context, the reintroduction of time-of-flight (TOF) PET may play an important role [10, 47]. PET systems with TOF capabilities are currently commercially available for clinical practice $[10,47]$. In TOF PET, the actual time difference in the arrival of the two annihilation protons at the detectors is recorded [10, 47]. The TOF information is incorporated directly into the reconstruction algorithm permitting some combination of faster scanning, improved SNR, or improved spatial resolution [10, 47].

Another very important issue is the fact that several tumors exhibit a maximum FDG uptake well beyond 60 min after FDG administration while surrounding normal tissues show a decline in FDG uptake with time [48-50]. Therefore, lesion-to-background contrast can be considerably increased at delayed PET imaging [48-50], thereby increasing detectability of the unknown primary tumor. It should be emphasized that especially in CUP, in which the primary tumor may be of small size and exhibit low FDG uptake, any improvement in sensitivity of the technique is crucial. Thus, it may be recommended to routinely use delayed PET imaging in this specific patient population. Of note, to the best of our knowledge, no study has investigated the promising use of delayed PET imaging in CUP yet.

Another issue worthy of mention is the use of dynamic FDG PET studies and PET parametric images which can be particularly useful for the detection and characterization of 
small lesions in the oropharynx, lung, liver, or pancreas [51]. This is presently an area of research and the search is on by several research centers to define a reliable and robust parametric imaging algorithm for dynamic PET studies as well as finding out the correct data reduction methods that can facilitate interpretation and substantiate visual analysis of large image sequences and at the same time can preserve important information [52, 53]. Despite its quantitative utility, dynamic PET imaging has been confined primarily to research centers and has not been in use in a busy clinical setting mainly because it is technically demanding.

Another promise for the future is the development of whole-body PET/MRI systems [54]. Although several technological difficulties have to be solved for designing a fully integrated whole-body PET/MRI system (including the issues of electromagnetic interference between the two systems and MRI-based attenuation correction), and its high (development) costs have to be taken into account, it theoretically offers several advantages over a combined $\mathrm{PET} / \mathrm{CT}$ system [54], which may also be of interest to patients with CUP. First, a fully integrated PET/MRI system allows true simultaneous scanning (instead of sequential scanning with PET/CT), thereby minimizing the mismatch between functional (PET) and anatomical (MRI) data sets [54]. Second, the superior soft tissue contrast of MRI and the possibility to acquire a variety of tissue contrasts may improve primary tumor detection and localization [54]. Especially in several body regions, including brain, liver, other soft tissue regions, and bone marrow, MRI may outperform CT [54, 55]. Third, MRI allows functional tissue information to be obtained, which may aid in primary tumor detectability. In this respect, whole-body diffusion-weighted MRI (DWI), a rapidly evolving functional whole-body technique, may play an important role [56, 57]. DWI depicts areas with an impeded diffusion, such as occurs in many malignant tumors. Similar to FDG PET, DWI offers a high lesion-tobackground contrast, making it a sensitive tool for the detection of lesions [56, 57]. The utility of whole-body DWI in patients with CUP was recently reported [58]. Advantages of DWI over FDG PET are its higher spatial resolution, and its superior ability in evaluating the urinary tract, where normal FDG accumulation may obscure lesions. Furthermore, apparent diffusion coefficient (ADC) measurements (a quantitative measure of diffusion in biological tissue) may allow characterization of suspicious lesions $[56,57,59]$. On the other hand, DWI also shows several normal structures with an impeded diffusion, and ADC measurements of benign and malignant lesions may overlap $[56,57,59]$. Thus, both FDG PET and DWI have their strengths and weaknesses, but a combination of both modalities may outperform either one of them alone; such a concept can be realized when PET/MRI systems become clinically available.

Finally, another promising development is the use of molecular technology that allows large-scale gene expression profiling. Several gene expression assays have been validated using samples from primary tumors or known metastatic lesions. The application of such a molecular assay that evaluates the expression of multiple tissue typespecific gene markers using quantitative reverse transcriptase polymerase chain reaction has shown to be very useful to predict the origin of the primary tumor in CUP $[60,61]$. The combination of molecular technology and advanced imaging techniques is expected to play an important role in the diagnostic management of patients with CUP.

\section{Conclusion}

In conclusion, an appropriate use and interpretation of FDG $\mathrm{PET} / \mathrm{CT}$ is necessary to maximize its diagnostic performance in CUP. In the near future, an advanced multiparametric approach, including the use of FDG PET/CT or FDG PET/MRI, functional whole-body MRI techniques, and molecular technology that allows gene expression profiling, is expected to outperform the diagnostic yield of FDG PET/CT alone. Studies investigating the impact of FDG PET/CT and other advanced diagnostic tests on therapeutic management, survival, and cost-effectiveness in CUP are of crucial importance.

Open Access This article is distributed under the terms of the Creative Commons Attribution Noncommercial License which permits any noncommercial use, distribution, and reproduction in any medium, provided the original author(s) and source are credited.

\section{References}

1. Pavlidis N, Fizazi K. Carcinoma of unknown primary (CUP). Crit Rev Oncol Hematol 2009;69:271-8.

2. Haas I, Hoffmann TK, Engers R, Ganzer U. Diagnostic strategies in cervical carcinoma of an unknown primary (CUP). Eur Arch Otorhinolaryngol 2002;259:325-3.

3. Raber MN, Faintuch J, Abbruzzese JL, Sumrall C, Frost P. Continuous infusion 5-fluorouracil, etoposide and cisdiamminedichloroplatinum in patients with metastatic carcinoma of unknown primary origin. Ann Oncol 1991;2:519-20.

4. Rege S, Maass A, Chaiken L, Hoh CK, Choi Y, Lufkin R, et al. Use of positron emission tomography with fluorodeoxyglucose in patients with extracranial head and neck cancers. Cancer 1994;73:3047-58.

5. Rohren EM, Turkington TG, Coleman RE. Clinical applications of PET in oncology. Radiology 2004;231:305-32.

6. Roh JL, Kim JS, Lee JH, Cho KJ, Choi SH, Nam SY, et al. Utility of combined (18)F-fluorodeoxyglucose-positron emission tomography and computed tomography in patients with cervical metastases from unknown primary tumors. Oral Oncol 2009;45:218-24. 
7. Nassenstein K, Veit-Haibach P, Stergar H, Gutzeit A, Freudenberg $\mathrm{L}$, Kuehl H, et al. Cervical lymph node metastases of unknown origin: primary tumor detection with whole-body positron emission tomography/computed tomography. Acta Radiol 2007;48:1101-8.

8. Freudenberg LS, Fischer M, Antoch G, Jentzen W, Gutzeit A, Rosenbaum SJ, et al. Dual modality of $18 \mathrm{~F}$-fluorodeoxyglucosepositron emission tomography/computed tomography in patients with cervical carcinoma of unknown primary. Med Princ Pract 2005;14:155-60.

9. Gutzeit A, Antoch G, Kühl H, Egelhof T, Fischer M, Hauth E, et al. Unknown primary tumors: detection with dual-modality PET/ CT_-initial experience. Radiology 2005;234:227-34.

10. Mawlawi O, Townsend DW. Multimodality imaging: an update on PET/CT technology. Eur J Nucl Med Mol Imaging 2009;36(Suppl 1): S15-29.

11. Kapoor V, McCook BM, Torok FS. An introduction to PET-CT imaging. Radiographics 2004;24:523-43.

12. Kwee TC, Kwee RM. Combined FDG-PET/CT for the detection of unknown primary tumors: systematic review and meta-analysis. Eur Radiol 2009;19:731-44.

13. Hany TF, Heuberger J, von Schulthess GK. Iatrogenic FDG foci in the lungs: a pitfall of PET image interpretation. Eur Radiol 2003;13:2122-7.

14. El-Haddad G, Alavi A, Mavi A, Bural G, Zhuang H. Normal variants in [18F]-fluorodeoxyglucose PET imaging. Radiol Clin North Am 2004;42:1063-81.

15. Fischbach F, Knollmann F, Griesshaber V, Freund T, Akkol E, Felix R. Detection of pulmonary nodules by multislice computed tomography: improved detection rate with reduced slice thickness. Eur Radiol 2003;13:2378-83.

16. Al-Brahim N, Ross C, Carter B, Chorneyko K. The value of postmortem examination in cases of metastasis of unknown origin-20-year retrospective data from a tertiary care center. Ann Diagn Pathol 2005;9:77-80.

17. Blaszyk H, Hartmann A, Bjornsson J. Cancer of unknown primary: clinicopathologic correlations. APMIS 2003;111:1089-94.

18. Mayordomo JI, Guerra JM, Guijarro C, García-Prats MD, Gómez A, López-Brea $\mathrm{M}$, et al. Neoplasms of unknown primary site: a clinicopathological study of autopsied patients. Tumori 1993;79:321-4.

19. Le Chevalier T, Cvitkovic E, Caille P, Harvey J, Contesso G, Spielmann M, et al. Early metastatic cancer of unknown primary origin at presentation. A clinical study of 302 consecutive autopsied patients. Arch Intern Med 1988;148:2035-9.

20. Fencl P, Belohlavek O, Skopalova M, Jaruskova M, Kantorova I, Simonova K. Prognostic and diagnostic accuracy of [18F]FDG$\mathrm{PET} / \mathrm{CT}$ in 190 patients with carcinoma of unknown primary. Eur J Nucl Med Mol Imaging 2007;34:1783-92.

21. Ay MR, Zaidi H. Assessment of errors caused by X-ray scatter and use of contrast medium when using CT-based attenuation correction in PET. Eur J Nucl Med Mol Imaging 2006;33:1301-13.

22. Namasivayam S, Kalra MK, Torres WE, Small WC. Adverse reactions to intravenous iodinated contrast media: a primer for radiologists. Emerg Radiol 2006;12:210-5.

23. Hainsworth JD, Greco FA. Treatment of patients with cancer of an unknown primary site. N Engl J Med 1993;329:257-63.

24. Fukui MB, Blodgett TM, Snyderman CH, Johnson JJ, Myers EN, Townsend DW, et al. Combined PET-CT in the head and neck: part 2. Diagnostic uses and pitfalls of oncologic imaging. Radiographics 2005;25:913-30.

25. Metser U, Even-Sapir E. Increased (18)F-fluorodeoxyglucose uptake in benign, nonphysiologic lesions found on whole-body positron emission tomography/computed tomography (PET/CT): accumulated data from four years of experience with PET/CT. Semin Nucl Med 2007;37:206-22.
26. Shim SS, Lee KS, Kim BT, Choi JY, Chung MJ, Lee EJ. Focal parenchymal lung lesions showing a potential of false-positive and false-negative interpretations on integrated PET/CT. AJR Am J Roentgenol 2006;186:639-48.

27. Wittram C, Scott JA. 18F-FDG PET of pulmonary embolism. AJR Am J Roentgenol 2007;189:171-6.

28. Pentheroudakis G, Briasoulis E, Pavlidis N. Cancer of unknown primary site: missing primary or missing biology? Oncologist 2007; 12:418-25.

29. van de Wouw AJ, Jansen RL, Speel EJ, Hillen HF. The unknown biology of the unknown primary tumour: a literature review. Ann Oncol 2003;14:191-6.

30. Sève P, Billotey C, Broussolle C, Dumontet C, Mackey JR. The role of 2-deoxy-2-[F-18]fluoro-D-glucose positron emission tomography in disseminated carcinoma of unknown primary site. Cancer 2007;109:292-9.

31. Rusthoven KE, Koshy M, Paulino AC. The role of fluorodeoxyglucose positron emission tomography in cervical lymph node metastases from an unknown primary tumor. Cancer 2004;101:2641-9.

32. Delgado-Bolton RC, Fernández-Pérez C, González-Maté A, Carreras JL. Meta-analysis of the performance of 18F-FDG PET in primary tumor detection in unknown primary tumors. J Nucl Med 2003;44:1301-14.

33. Werner JA, Dünne AA, Myers JN. Functional anatomy of the lymphatic drainage system of the upper aerodigestive tract and its role in metastasis of squamous cell carcinoma. Head Neck 2003;25:322-32.

34. Rosen EL, Eubank WB, Mankoff DA. FDG PET, PET/CT, and breast cancer imaging. Radiographics 2007;27(Suppl 1):S215-29.

35. Buchanan CL, Morris EA, Dorn PL, Borgen PI, Van Zee KJ. Utility of breast magnetic resonance imaging in patients with occult primary breast cancer. Ann Surg Oncol 2005;12:1045-53.

36. Schorn C, Fischer U, Luftner-Nagel S, Westerhof JP, Grabbe E. MRI of the breast in patients with metastatic disease of unknown primary. Eur Radiol 1999;9:470-3.

37. Kaya AO, Coskun U, Unlu M, Akdemir UO, Ozdemir NY, Zengin N, et al. Whole body 18 F-FDG PET/CT imaging in the detection of primary tumours in patients with a metastatic carcinoma of unknown origin. Asian Pac J Cancer Prev 2008;9:683-6.

38. Gladish GW, Choe DH, Marom EM, Sabloff BS, Broemeling LD, Munden RF. Incidental pulmonary emboli in oncology patients: prevalence, CT evaluation, and natural history. Radiology 2006;240:246-55.

39. Schapira DV, Jarrett AR. The need to consider survival, outcome, and expense when evaluating and treating patients with unknown primary carcinoma. Arch Intern Med 1995;155:2050-4.

40. Basu S, Alavi A. FDG-PET in the clinical management of carcinoma of unknown primary with metastatic cervical lymphadenopathy: shifting gears from detecting the primary to planning therapeutic strategies. Eur J Nucl Med Mol Imaging 2007;34:427-8.

41. Fletcher JW, Djulbegovic B, Soares HP, Siegel BA, Lowe VJ, Lyman GH, et al. Recommendations on the use of 18F-FDG PET in oncology. J Nucl Med 2008;49:480-508.

42. Hillner BE, Siegel BA, Liu D, Shields AF, Gareen IF, Hanna L, et al. Impact of positron emission tomography/computed tomography and positron emission tomography (PET) alone on expected management of patients with cancer: initial results from the National Oncologic PET Registry. J Clin Oncol 2008;26:2155-61.

43. Bruna C, Journo A, Netter F, Kaminsky MC, Dolivet G, Olivier P, et al. On the interest of PET with $18 \mathrm{~F}-\mathrm{FDG}$ in the management of cancer of unknown primary (CUP). Med Nucl 2007;31:242-9.

44. Wartski M, Le Stanc E, Gontier E, Vilain D, Banal A, Tainturier C, et al. In search of an unknown primary tumour presenting with cervical metastases: performance of hybrid FDG-PET-CT. Nucl Med Commun 2007;28:365-71. 
45. Fakhry N, Barberet M, Lussato D, Cammilleri S, Mundler O, Giovanni A, et al. Role of [18F]-FDG PET-CT in the management of the head and neck cancers. Bull Cancer 2006;93:1017-25.

46. Pelosi E, Pennone M, Deandreis D, Douroukas A, Mancini M, Bisi G. Role of whole body positron emission tomography/ computed tomography scan with $18 \mathrm{~F}$-fluorodeoxyglucose in patients with biopsy proven tumor metastases from unknown primary site. Q J Nucl Med Mol Imaging 2006;50:15-22.

47. Surti S, Kuhn A, Werner ME, Perkins AE, Kolthammer J, Karp JS. Performance of Philips Gemini TF PET/CT scanner with special consideration for its time-of-flight imaging capabilities. J Nucl Med 2007;48:471-80.

48. Kumar R, Dhanpathi H, Basu S, Rubello D, Fanti S, Alavi A. Oncologic PET tracers beyond [(18)F]FDG and the novel quantitative approaches in PET imaging. Q J Nucl Med Mol Imaging 2008;52:50-65.

49. Basu S, Kung J, Houseni M, Zhuang H, Tidmarsh GF, Alavi A. Temporal profile of fluorodeoxyglucose uptake in malignant lesions and normal organs over extended time periods in patients with lung carcinoma: implications for its utilization in assessing malignant lesions. Q J Nucl Med Mol Imaging 2009;53:9-19.

50. Sanz-Viedma S, Torigian DA, Parsons M, Basu S, Alavi A. Potential clinical utility of dual time point FDG-PET for distinguishing benign from malignant lesions: implications for oncological imaging. Rev Esp Med Nucl 2009;28:159-66.

51. Anzai Y, Minoshima S, Wolf GT, Wahl RL. Head and neck cancer: detection of recurrence with three-dimensional principal components analysis at dynamic FDG PET. Radiology 1999;212:285-90.

52. Pavlopoulos S, Thireou T, Kontaxakis G, Santos A. Analysis and interpretation of dynamic FDG PET oncological studies using data reduction techniques. Biomed Eng Online 2007;6:36.
53. Thireou T, Pavlopoulos S, Kontaxakis G, Santos A. Blind source separation for the computational analysis of dynamic oncological PET studies. Oncol Rep 2006;15:1007-12.

54. von Schulthess GK, Schlemmer HP. A look ahead: PET/MR versus PET/CT. Eur J Nucl Med Mol Imaging 2009;36(Suppl 1): S3-9.

55. Antoch G, Vogt FM, Freudenberg LS, Nazaradeh F, Goehde SC, Barkhausen J, et al. Whole-body dual-modality PET/CT and whole-body MRI for tumor staging in oncology. JAMA 2003;290:3199-206.

56. Kwee TC, Takahara T, Ochiai R, Katahira K, Van Cauteren M, Imai $\mathrm{Y}$, et al. Whole-body diffusion-weighted magnetic resonance imaging. Eur J Radiol 2009;70:409-17.

57. Kwee TC, Takahara T, Ochiai R, Nievelstein RA, Luijten PR. Diffusion-weighted whole-body imaging with background body signal suppression (DWIBS): features and potential applications in oncology. Eur Radiol 2008;18:1937-52.

58. Gu TF, Xiao XL, Sun F, Yin JH, Zhao H. Diagnostic value of whole body diffusion weighted imaging for screening primary tumors of patients with metastases. Chin Med Sci J 2008;23:145-50.

59. Mori T, Nomori H, Ikeda K, Kawanaka K, Shiraishi S, Katahira K, et al. Diffusion-weighted magnetic resonance imaging for diagnosing malignant pulmonary nodules/masses: comparison with positron emission tomography. J Thorac Oncol 2008;3:358-64.

60. Varadhachary GR, Talantov D, Raber MN, Meng C, Hess KR, Jatkoe T, et al. Molecular profiling of carcinoma of unknown primary and correlation with clinical evaluation. J Clin Oncol 2008;26:4442-8.

61. Horlings HM, van Laar RK, Kerst JM, Helgason HH, Wesseling $\mathrm{J}$, van der Hoeven JJ, et al. Gene expression profiling to identify the histogenetic origin of metastatic adenocarcinomas of unknown primary. J Clin Oncol 2008;26:4435-41. 